\title{
Como estruturar uma atividade para melhoria do ensino e do trabalho interprofissional em um hospital universitário?
}

Carla Suely Souza de Paula, Cesimar Severiano do Nascimento, Niethia Regina Dantas de Lira, Natalia Castro de Carvalho Schachnik Nogueira, Maria Fernanda de Oliveira Carvalho

\begin{abstract}
Resumo
Introdução: A Educação Interprofissional (EIP) pode ser definida como a situação em que dois ou mais profissionais aprendem juntos sobre, a partir do e um com o outro, visando proporcionar a melhoria da assistência ao paciente1. A EIP e as Práticas Colaborativas (PC) estão tendo um enfoque cada vez maior na educação dos profissionais na área de saúde, visto que promove a desejada atenção integral ao indivíduo e, portanto, a melhoria da assistência. Tal fato foi evidenciado por Anderson, que estudou grupos interprofissionais de saúde na comunidade e Gibson et al que relataram a inserção de outros profissionais na atenção ao paciente psiquiátrico. Holland et al2 descreveram a redução das internações hospitalares de pacientes com insuficiência cardíaca quando acompanhados por equipes multiprofissionais. Observava-se, de forma semelhante, a redução da mortalidade em pacientes críticos internados em unidade de terapia intensiva (UTI) que eram acompanhados por equipes multiprofissionais. Autores como Barr, Carpenter, Linston e Anderson ressaltaram a importância de introduzir esta prática durante a graduação, bem como forneceram estratégias para tal. $\mathrm{O}$ momento correto para a introdução da atividade se, no início do curso ou mais tardiamente, ainda é controverso. No Brasil o interesse sobre as Atividades Interprofissionais (AIPs) vem crescendo nos últimos anos, visto que a integralidade da assistência à saúde constitui um dos eixos prioritários do Sistema Único de Saúde (SUS). O cenário brasileiro, entretanto, mostra que a formação é, sobretudo, uniprofissional e que as iniciativas de EIP ainda são tímidas, fazendo referência principalmente às ações multiprofissionais, seja na graduação, pósgraduação latu senso e, mais recentemente, referentes às atividades optativas extracurriculares como o Programa de Educação pelo Trabalho para a Saúde (PET-Saúde). Tais atividades frequentemente envolvem tutores da rede de saúde, estudantes e docentes da saúde, o Telessaúde, a Educação Profissional, o Programa de Formação de Profissionais de Nível Médio para Saúde (PROFAPS) e a Universidade Aberta do Sistema Único de Saúde (UNASUS).3 A relevância do Trabalho Interprofissional (TIP) foi destacada na recente publicação das Diretrizes Curriculares Nacionais para os Cursos de Medicina (2014)4, onde se observa que deve prevalecer o trabalho interprofissional em equipe, o desenvolvimento de relação horizontal, compartilhada, respeitando-se as necessidades e desejos da pessoa cuidada, a família e a comunidade. Um dos objetivos da formação é aprender interprofissionalmente com base na reflexão sobre a própria prática e pela troca de saberes com profissionais da área da saúde e de outras áreas do conhecimento, para a orientação, identificação e discussão dos problemas, estimulando o aprimoramento da colaboração e da qualidade da atenção à saúde. A despeito da importância do TIP e da EIP para a atenção integral, melhoria da segurança do paciente e do processo de trabalho das equipes, a atuação interpofissional é ainda bastante desafiadora em função da fragilidade das competências relacionadas às práticas colaborativas como a comunicação interpessoal5. Apesar da importância na alta complexidade e em especial, na abordagem ao paciente cardiopata, são escassas as experiências formais de ensino do TIP nas enfermarias do HUOL. O contato com os outros profissionais é realizado, habitualmente, através de uma abordagem individual, no formato de interconsultas, sem haver o compartilhamento ou troca de informações com o grupo, o que muitas vezes, proporciona grande dificuldade na comunicação entre as equipes, comprometendo a assistência. Assim, reconhecendo a relevância do
\end{abstract}


TIP na assistência ao paciente cardiopata e a necessidade de adequação da formação voltada para a inserção da EIP e a realidade do HUOL, surgiram algumas questões: Será que pacientes internados no serviço de cardiologia do HUOL seriam favorecidos por essa prática? Seria possível, baseados nos modelos encontrados na literatura, elaborar uma estratégia de TIP e EIP factível a realidade de um hospital terciário, inserindo uma estratégia de EIP na formação para melhoria no aprendizado dos estudantes? Objetivos $\mathrm{O}$ objetivo deste estudo é descrever o processo de implantação da Reunião Interprofissional da Cardiologia (RIC) como estratégia de ensino da educação interprofissional na graduação e pós-graduação da Universidade Federal do Rio grande do Norte (UFRN). Métodos Trata-se de um estudo tipo pesquisa-ação, qualitativo, exploratório e prospectivo, realizado de março de 2013 a novembro de 2014, no serviço de cardiologia do Hospital Universitário Onofre Lopes (HUOL), envolvendo profissionais da área da saúde do HUOL e estudantes de graduação e pós- graduação dos cursos de saúde da Universidade Federal do Rio grande do Norte (UFRN), desenvolvido em três etapas: Planejamento, implantação e avaliação da RIC. Essa foi feita um questionário semiestruturado online, utilizando a plataforma SurveyMonkey®, contendo questões fechadas com escala de Likert de cinco pontos, além de grupo focal para analisar a percepção dos participantes quanto a nova estratégia interprofissional. A análise dos dados foi descritiva, a partir dos resultados obtidos com o questionário. Para a análise qualitativa, foi realizada a análise de conteúdo, conforme a temática categorial de Bardin. O estudo foi analisado e aprovado pelo Comitê de Ética em Pesquisa do Hospital Universitário Onofre Lopes - CEP/HUOL, parecer no 666.965 e registro CAAE no 16420513.7.0000.5292. Resultados Após dois meses de planejamento, a etapa de implementação teve início, sendo realizadas 60 reuniões, discutido um caso clinico por sessão, envolvendo 1357 participantes. Após apresentação de cada caso, houve discussão interprofissional, ressaltando a especificidade de cada profissional para melhoria do cuidado integral ao paciente em questão. A liderança foi alternada de acordo com a necessidade individual do caso a ser discutido. Os grupos focais foram avaliados pela Análise Categorial de Bardin onde emergiram cinco categorias: Reconhecimento da participação em atividades interprofissionais prévias; Visão conceitual de atividades interprofissionais; Impactos da RIC na assistência; Contribuições da RIC na formação e Desafios da manutenção da RIC. Considerações finais (conclusões) $\mathrm{O}$ processo de planejamento e implantação da RIC atingiu os objetivos. Foi observado, pela análise dos grupos focais, que a RIC é uma estratégia que impacta positivamente tanto na assistência quanto no ensino, em especial nas residências em saúde. Em função da experiência exitosa da RIC, nos anos subsequentes houve fortalecimento e estruturação do serviço de cardiologia, que passou a ser reconhecido como equipe CardioHUOL. Novos profissionais foram integrados permanentemente a equipe interprofissional propiciando o surgimento da Residência Multiprofissional em Cardiologia. A visita interprofissional a beira do leito realizada semanalmente foi implantada e atividades similares foram iniciadas no serviço de Oncologia da instituição.

Descritores: Equipe de Assistência ao Paciente, Cardiologia, Relações interprofissionais, Educação Médica Pós-graduação 